\title{
Satisfactions and frustrations of a volunteer tele-reader for the World Federation of Pediatric Imaging
}

\author{
Cicero T. Silva
}

Received: 11 November 2013 / Accepted: 10 March 2014

(C) Springer-Verlag Berlin Heidelberg 2014

I first got involved with the World Federation of Pediatric Imaging (WFPI) in August 2012 through its outreach program. Just a few weeks after the first e-mail contact I was reading pediatric radiographs from Khayelitsha Hospital in South Africa. Images were sent as JPEG files through e-mail by a local radiographer (WFPI now uses a DICOM server). I got to report about 15 radiographs a month. The pathology varied, including pneumonias, congenital heart disease and trauma (Fig. 1). Because the hospital has no radiologist, my emailed reports were forwarded to the referring clinicians.

About 50 pediatric radiologists from all continents have enlisted in the WFPI outreach program. The program provides a unique channel through which those radiologists volunteer their imaging expertise, truly impacting the diagnosis and management of sick children from low-resource areas.

The can-do attitude of WFPI leaders and staff was contagious, and 2 months later I was embracing another project, the creation of a tele-reading site in South Asia, where we would be providing opinions on pediatric cases referred by the local radiologist. We faced several challenges in setting up this site, many related to the geographical distances among the many players. Meetings were difficult to schedule because of time zone differences, thus communications happened primarily through e-mail. Multiple people replying to the same e-mails meant ideas were getting lost in the threads as simultaneous conversations took diverging courses. A tele-reading computer platform was created, but it took a while to get the referring site and WFPI volunteers to install the software. When we were finally ready to start, the referrals didn't come! When WFPI sent inquiring e-mails, the replies from the site were reticent and apathetic. Was this caused by politics on the ground? Were local radiologists threatened? We weren't sure. The experience was quite frustrating, but we learned from it.

\section{T. Silva $(\square)$}

Department of Diagnostic Imaging,

Yale University School of Medicine, 333 Cedar St.,

P.O. Box 208042, New Haven, CT 06520, USA

e-mail: cicero.silva@yale.edu

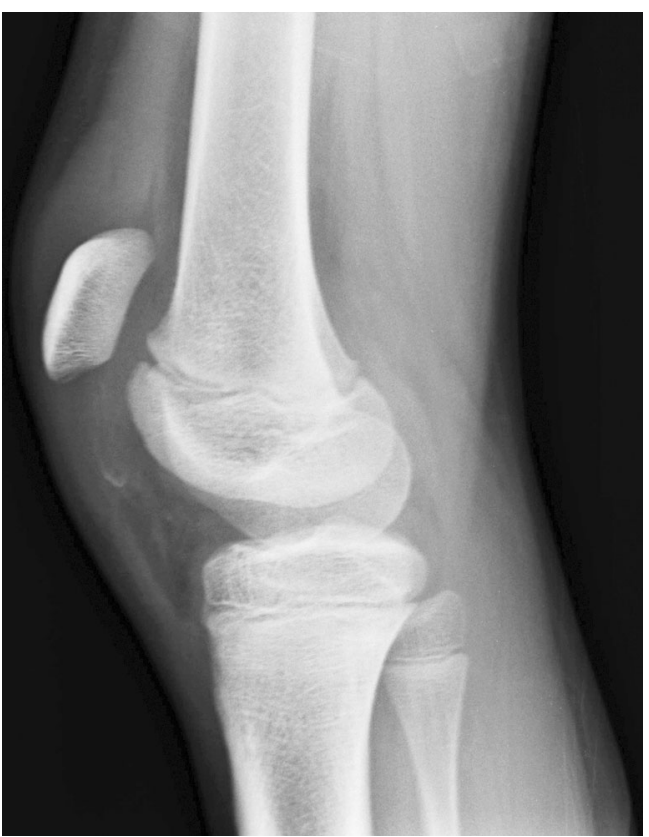

Fig. 1 Patellar sleeve avulsion in an 11-year-old boy from South Africa who had a painful knee after a fall from a height. Lateral shoot-through right knee radiograph, reviewed as normal by the local emergency clinician. The radiograph shows the patella situated more superior than expected. That is associated with buckling of the quadriceps tendon, a suprapatellar joint effusion, and a faint V-shape density along the expected position of the inferior patellar pole. Following the WFPI read of patellar sleeve avulsion the patient was referred to a tertiary hospital and treated in an orthopedics department

We have learned from that setup failure that the WFPI outreach efforts need to be matched by local partners who are as keen on the idea. The local radiologist, if there is one, needs to be particularly engaged, perceiving WFPI not as a competitor but as a powerful source of subspecialist expertise that adds value to the local radiology department.

Currently, WFPI outreach teams provide support to sites in India and South Africa, and in partnership with Medecins Sans Frontieres to sites in Cambodia, Central African Republic, Malawi and Tajikistan.

Conflict of interest None 